\title{
Well Control Optimization of Waterflooding Oilfield Based on Deep Neural Network
}

\author{
Lihui Tang $\mathbb{D}^{1,2}$ Junjian Li $\mathbb{D}^{1,2}$ Wenming Lu $\mathbb{D}^{3},{ }^{3}$ Peiqing Lian $\mathbb{D}^{3},{ }^{3}$ Hao Wang $\mathbb{D},{ }^{1,2}$ \\ Hanqiao Jiang $\mathbb{D},{ }^{1,2}$ Fulong Wang $\mathbb{D}^{1,2}$ and Hongge Jia $\mathbb{C}^{4}$ \\ ${ }^{1}$ College of Petroleum Engineering, China University of Petroleum (Beijing), Beijing 102249, China \\ ${ }^{2}$ State Key Laboratory of Petroleum Resources and Prospecting, China University of Petroleum (Beijing), Beijing 102249, China \\ ${ }^{3}$ SINOPEC Petroleum Exploration and Production Research Institute, Beijing 100083, China \\ ${ }^{4}$ Aktobe Corporation, PetroChina International (Kazakhstan), Beijing 100011, China
}

Correspondence should be addressed to Junjian Li; junjian@126.com

Received 22 August 2020; Revised 23 February 2021; Accepted 14 March 2021; Published 7 April 2021

Academic Editor: Guanglong Sheng

Copyright (c) 2021 Lihui Tang et al. This is an open access article distributed under the Creative Commons Attribution License, which permits unrestricted use, distribution, and reproduction in any medium, provided the original work is properly cited.

A well control optimization method is a key technology to adjust the flow direction of waterflooding and improve the effect of oilfield development. The existing well control optimization method is mainly based on optimization algorithms and numerical simulators. In the face of larger models, longer optimization periods, or reservoir models with a large number of optimized wells, there are many optimization variables, which will cause algorithm convergence difficulties and optimization costs. The application effect is not good because of the problems of time length, few comparison schemes, and only fixed control frequency. This paper proposes a new method of a well control optimization method based on a multi-input deep neural network. This method takes the production history data of the reservoir as the main input and the saturation field as the auxiliary input and establishes a multi-input deep neural network for learning, forming a production dynamic prediction model instead of conventional numerical simulators. Based on the production dynamic prediction model, a series of model generation, production prediction, comparison, and optimization are carried out to find the best production plan of the reservoir. The calculation results of the examples show that (1) compared with the single-input production dynamic prediction model, the production dynamic prediction model based on multiple inputs has better prediction accuracy, and the results are close to the calculation results of the conventional numerical simulator; (2) the well control optimization method based on the multipleinput deep neural network has a fast optimization speed, with many comparison schemes and good optimization effect.

\section{Introduction}

As the development of waterflooding oilfields progresses, due to the influence of reservoir heterogeneity, the directionality of waterflooding increases and the difference in plane production increases, which gradually weakens the effect of water control and oil increase, forming a large area of remaining oil retention. Through the optimization of the production system, the flow direction of waterflooding can be adjusted to increase the potential of remaining oil and promote the effect of reservoir development. And for the oilfield site, it is often not feasible to adopt a constant oilfield production strategy, because it requires unrealistic bottom hole pressure, which requires us to set up a set of changeable production dynamic programs [1].

Over the past decades, a large number of experts and scholars have conducted research on this, which can be summarized into three categories: (1) Based on the reservoir engineering formula method. This method combines the material balance and trend fitting method to establish the production dynamic prediction formula, then calculates the production dynamics of different injection-production schemes, obtains the objective function value (such as net present value and cumulative oil production), and then compares and selects the best [2-4]. But because reservoir development is affected by multiple factors, and this method has ideal assumptions, it 
leads to problems such as poor accuracy of results and narrow method adaptability. (2) Based on reservoir numerical simulation methods: this method first establishes a numerical simulation model based on the reservoir and fluid data provided by the oilfield, then uses the orthogonal experimental design method to set up multiple sets of different injection and production plans, and finally compares and selects the best based on parameters such as net present value [5-9]. However, in order to consider the impact of reservoir heterogeneity, the grids are often finely divided. If multiple numerical simulation programs are run, it will consume a lot of time and manpower. In the process of comparing experimental schemes, the value range of factors is limited, only a few or dozens of schemes can be compared, and it is difficult to obtain the real optimal injection-production scheme. At the same time, the current method is basically static optimization, and the result of dynamic optimization cannot be obtained. (3) Method based on optimization theory: this method applies optimization theory to find the best plan, uses reservoir numerical simulation to predict the production performance of the plan, then calculates the value of the objective function, and finally obtains the best injectionproduction plan [10-18]. This method can realize dynamic optimization and can compare more sets of injectionproduction schemes. Scholars can also achieve multiobjective optimization based on this method and achieve more rational decision-making $[19,20]$. However, this method relies on numerical simulation calculation, which also has the problem of time-consuming. And as the number of wells increases and the frequency of regulation increases, the number of optimization variables will greatly increase, which is likely to cause dimensionality disasters. This type of method is more often used for well pattern optimization [21, 22]. At present, this method is still difficult to solve the problem of obtaining the optimal control frequency.

In recent years, deep learning has provided new ideas and methods for oilfield development. Many scholars use longand short-term memory (LSTM) to predict production dynamics, pressure, and other time series data [23-29]. Based on the existing problems of the existing well control optimization methods and the inspiration of deep learning, this article establishes a new method of production dynamic prediction based on deep neural networks. This method has a faster optimization speed and more comparison schemes and can find the optimal control frequency. This method establishes a production dynamic prediction model based on a multi-input deep neural network instead of conventional numerical simulation software to calculate production dynamics, which greatly reduces the model production dynamic prediction time. Subsequent comparison schemes are randomly generated under the constraints of reservoir engineering, production performance prediction models are used to quickly predict production performance, and then the objective function value of each scheme is calculated to compare and optimize the optimal scheme. In addition, this method takes the control frequency as the optimization parameter, and finally, after a large number of schemes are optimized, the control frequency of the optimal scheme is close to the optimal control frequency.
The core of this method is to establish a production dynamic prediction model based on a deep neural network to replace conventional numerical simulators. In establishing the production dynamic prediction model, former scholars found that the use of traditional production prediction methods, such as the water drive curve method, the decline curve method, and Weng's prediction method, has low prediction accuracy, especially in high water-cut oilfields [3034]. In order to solve the shortcomings of traditional forecasting methods, intelligent algorithms have also been introduced into oilfield production forecasts. Because the output is a sequential problem, in recent years, scholars have begun to use the long- and short-term memory network LSTM algorithm. However, oilfield production data has a strong phased nature. In oil reservoir engineering, it is divided into a period of rising production, a period of stable production, and a period of declining production, with large fluctuations. Using conventional long- and short-term memory neural networks to predict oilfield production indicators, there will be a problem of poor generalization of the neural network, low prediction accuracy, and even negative values for the predicted output, with large deviations [35], especially when the sample size is small; this phenomenon is more prominent [36-38]. In view of the poor generalization of the LSTM neural network, scholars reduce the error by optimizing the neural network architecture. Mao [39] proposed a long text sentiment analysis method based on the attention double-layer LSTM; Peng et al. [40] used double LSTM layers to adjust the parameters to improve the accuracy of sentence generation. Because the volume of data that can be obtained for reservoir development is limited, it is necessary to introduce reservoir engineering constraint input to obtain higher prediction accuracy with a smaller volume of data. Based on the above two considerations, this article will propose a deep neural network structure based on multiple inputs to optimize the LSTM neural network, so that reservoir production predictions can be taken into account in oilfield development and improve the reliability and accuracy of the simulator.

This article will first introduce the core issue of the method, namely, the establishment of a production dynamic prediction model based on multi-input deep neural networks; secondly, this article will introduce how to optimize production dynamics based on the production dynamic prediction model; thirdly, this article will apply the well control optimization method to optimize the actual reservoir and conducts related discussions and finally draws a conclusion.

\section{Neural Network Structure of Production Dynamic Prediction Model}

Production dynamic prediction is a time series prediction problem, and a long- and short-term memory neural network [41] (long- and short-term memory, LSTM) has excellent accuracy in time series prediction problems and has been widely used in speech recognition [42], network flow prediction [43], predrilling log curve prediction [44], and other fields. However, due to the staged characteristics of oilfield production, there will be a problem of poor generalization of neural networks. This article will optimize the neural 
network structure and propose a multi-input neural network structure suitable for oilfield production data prediction. The multi-input neural network structure is based on production dynamic data and saturation field data, so that the trained simulator can have a good prediction effect on the phased production characteristics of the oilfield, thereby improving the prediction accuracy of the production dynamic prediction model.

Because the production dynamic data is time series data, in deep learning, the most suitable deep neural network algorithm is the recurrent neural network algorithm (RNN), but the RNN has the problem of disappearing gradient. The long- and short-term memory network (LSTM) solves the problem of RNN gradient disappearance and realizes longterm memory ability by improving the hidden layer unit structure of the recurrent neural network. After continuous testing by predecessors, it is proved that it has higher prediction accuracy than the RNN and is widely used. Therefore, the key algorithm used in the deep neural network established in this article is the LSTM algorithm.

\subsection{From RNN to LSTM}

2.1.1. Recurrent Neural Network. The recurrent neural network (RNN) can transfer the state information of the last time to the current time, that is, the state of the current time is the result of the joint action of the input of the current time and the information of the last time. It has outstanding performance in the field of natural language processing and is widely used in machine translation and text recognition. Its network structure is shown in Figure 1.

Assuming that the input time series data is $X=\left\{x_{0}, x_{1}\right.$, $\left.x_{2}, \cdots, x_{t}, \cdots, x_{n}\right\}$, and the output time series data is $O=\left\{o_{0}\right.$ $\left., o_{1}, o_{2}, \cdots, o_{t}, \cdots, o_{n}\right\}$, then the RNN calculation process is

$$
\begin{aligned}
& s_{t}=f\left(U x_{t}+W s_{t-1}+b\right), \\
& o_{t}=\operatorname{soft} \max \left(V s_{t}\right) .
\end{aligned}
$$

Among them, $s$ is the value of the hidden layer; $U$ is the weight matrix of the input layer; $o$ is the value of the output layer; $V$ is the weight matrix of the hidden layer to the output layer; $W$ is the previous value of the hidden layer as the weight matrix of this input; $f$ is generally a nonlinear activation function; $b$ is a bias vector.

However, this method has the problem that the gradient disappears, that is, the state at time $t-3$ has no significant effect on the state at time $t$, which is equivalent to the network ignoring the state before time $t-3$ during training. So Hochreiter and Schmidhuber proposed the LSTM network [41], and Hochreiter subsequently demonstrated the ability of LSTM to avoid a vanishing gradient problem [45]. It has become one of the most popular algorithms in recurrent neural networks.

2.1.2. Long- and Short-Term Memory Network (LSTM). The most important difference between LSTM and RNN is the hidden layer structure. The long-term memory ability is obtained by replacing the RNN as a unit with the LSTM as

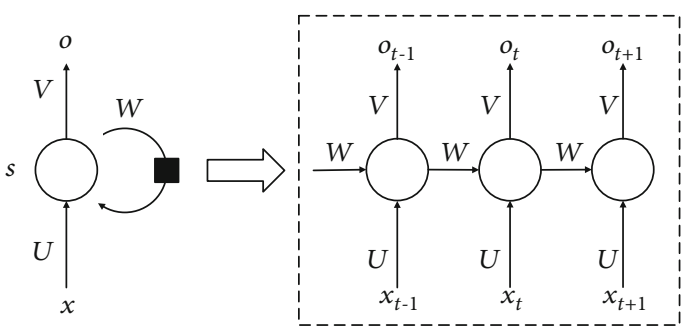

FIgURE 1: Schematic diagram of the recurrent neural network.

a unit. The LSTM hidden layer neural unit is shown in Figure 2.

LSTM deletes or adds information through the gate structure, realizes the forgetting and memory functions of the neural unit, establishes long-term dependence between time series data, and realizes the transmission of information in different time spans. An LSTM hidden layer unit has three gate structures, namely, the forget gate, input gate, and output gate. The LSTM calculation process is as follows:

$$
\begin{aligned}
& f_{t}=\sigma\left(W_{f} \cdot\left[h_{t-1}, x_{t}\right]+b_{f}\right), \\
& i_{t}=\sigma\left(W_{i} \cdot\left[h_{t-1}, x_{t}\right]+b_{f i}\right), \\
& \tilde{C}_{t}=\tanh \left(W_{C} \cdot\left[h_{t-1}, x_{t}\right]+b_{C}\right), \\
& C_{t}=f_{t} * C_{t-1}+i_{t} * \tilde{C}_{t}, \\
& o_{t}=\sigma\left(W_{o} \cdot\left[h_{t-1}, x_{t}\right]+b_{o}\right), \\
& h_{t}=o_{t} * \tanh \left(C_{t}\right) .
\end{aligned}
$$

Among them, $\sigma$ is an activation function, generally a sigmoid function; $W_{f}$ and $b_{f}$ are the connection weight matrix and bias vector of the forgetting gate, respectively; $W_{i}$ and $b_{i}$ are the connection weight matrix and offset vector of the input data and input gate; $W_{C}$ and $b_{C}$ are the connection weight matrix and offset vector of the input data and the state of the temporary unit; $W_{o}$ and $b_{o}$ are the connection weight matrix and offset vector of the output gate; $h_{t-1}$ is the hidden layer value at the previous time; $x_{t}$ is the current time input value; $f_{t}$ is the value of the forget gate; it is the value of the memory gate; tanh is the activation function; $C_{t}$ is the unit state at the current time; $C_{t-1}$ is the unit state at the previous time; $\tilde{C}_{t}$ is the temporary unit state; $o_{t}$ is the output gate value.

2.2. Optimized Multi-Input Deep Neural Network Structure. This paper establishes a multi-input deep neural network structure to replace the conventional single-input deep neural network structure. Combining with the characteristics of oilfield production, the main input of the multi-input deep neural network is set to the production history data of each well at each time, and the auxiliary input is the oil saturation field at each time, so that the optimized network model can predict more accurately. The established multi-input production dynamic prediction model can predict the production 


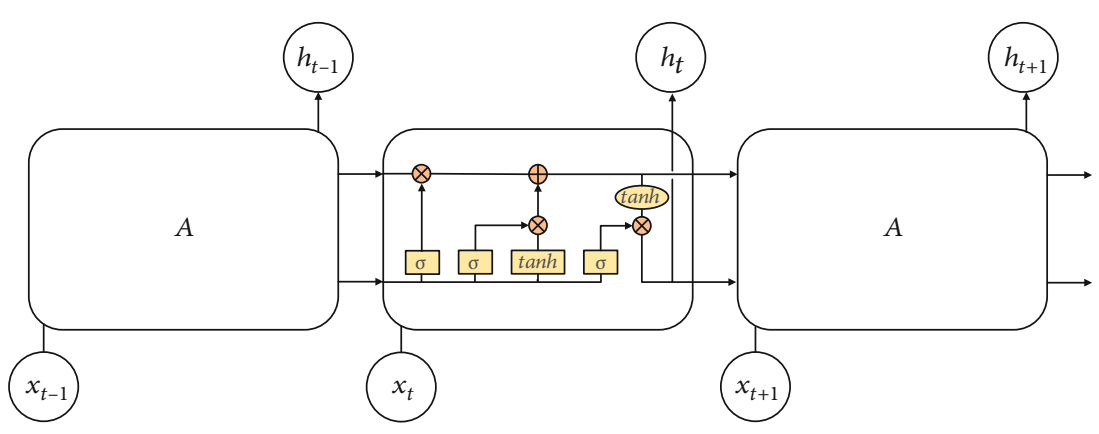

FIGURE 2: LSTM hidden layer neural unit.

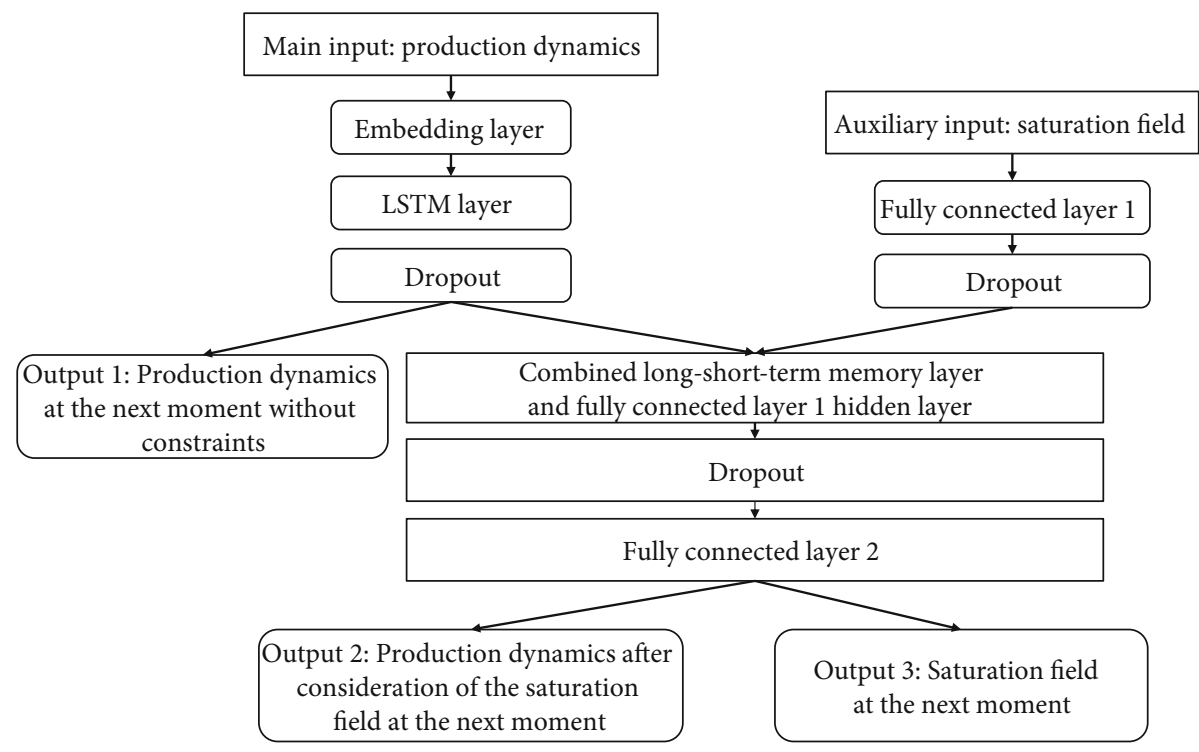

FIgURE 3: Schematic diagram of the deep neural network structure.

dynamic results of oilfields with large fluctuations and stage characteristics. The neural network link method is to first combine the main input and the LSTM algorithm to obtain hidden layer 1 , then combine the auxiliary input and the fully connected layer to obtain hidden layer 2, and then combine the two hidden layers into a new hidden layer. Afterwards, the final output is obtained through multiple fully connected layers and random inactivation layers. The structure of a multi-input deep neural network is shown in Figure 3.

The important reasons why the neural network selects the oil saturation field as the auxiliary input are as follows: (1) the oil saturation field is a comprehensive performance of the current production situation of the reservoir and has the most direct correlation with the phased fluctuation characteristics of reservoir production. Therefore, the selection of the oil saturation field for auxiliary input can help solve the problem that the neural network cannot predict the phased fluctuation characteristics of the oilfield production dynamics; (2) another important reason is that the oil saturation field is the same as the historical production data. The same time series data, after neural network training, can learn the correlation between oil saturation field and production dynamics at each moment, while other attribute fields, such as porosity and permeability, are fixed values. If it is used as an auxiliary input, the neural network cannot learn the corre- lation between the dynamic production data and the different production stages of the oilfield but affects the accuracy.

The neural network sets up multiple dropout layers to avoid the problem of reduced generalization ability caused by overfitting. This method was proposed by Srivastava et al. [46]. Its core idea is that in the training process of a neural network, the network updates some nodes according to Bernoulli probability, and some nodes are randomly discarded without updating.

\section{Production Dynamic Optimization Method}

The principle of the well control optimization methods is as follows: read production dynamic data and oil saturation field data from the numerical simulation model, form training and test data sets through data processing, and use these data to establish a production dynamic prediction model based on a multi-input deep neural network. Then, randomly generate injection-production schemes with different injection-production parameters, apply the production dynamic prediction model to predict the production dynamics of each scheme, calculate the objective function value, and select the scheme corresponding to the largest objective function as the optimal scheme. The flow chart is shown in Figure 4. 


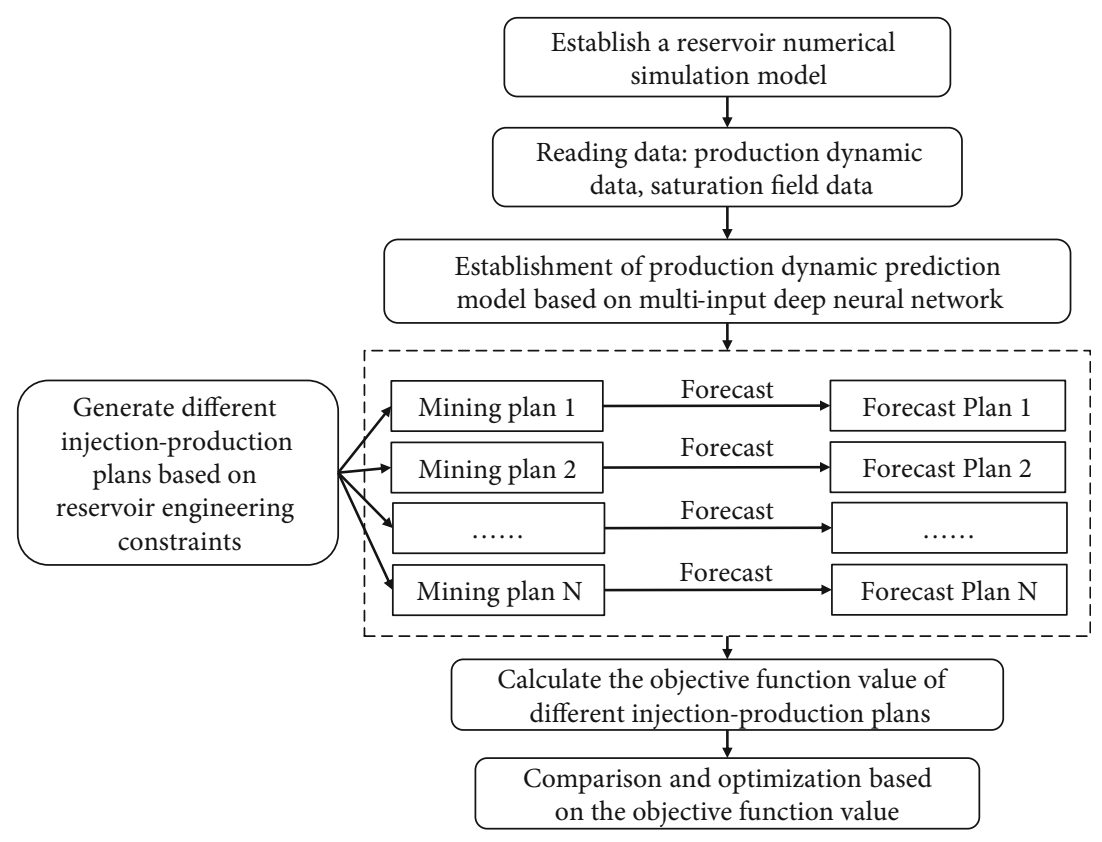

Figure 4: Flow chart of the production dynamic optimization model.

3.1. Numerical Model Establishment and Data Collection. First, establish a numerical simulation based on the geological data and fluid data of the reservoir, and then read the production history data and oil saturation field data at each moment in the model. The model assumes that there are $N$ wells, producing $T$ days. Then, the production dynamics as the main input and the saturation field data of the auxiliary input are as follows:

(1) Production dynamic data: assuming that it is set on day $t$, and $q_{t}$ consists of water injection volume of all water injection wells, oil production volume, and liquid production volume of all production wells, the data table of the production dynamics as the main input is $Q=\left\{q_{0}, q_{1}, \cdots q_{t} \cdots, q_{n-1}\right\}$. The production dynamics of all wells are synthesized into a data matrix as input at one time, and the neural network structure learning can map the interwell connection and production relations of multiple wells, which is closer to the actual production of the reservoir

(2) Saturation field data: the saturation data of the $5 \times 5$ grid set around the $i$ th well on the $t$ th day form a vector $s_{t, i}$ in the order shown in Figure 5

Then, the saturation vector composed of all wells on day $i$ is $S_{t}=\left\{s_{t, 1}, s_{t, 2}, \cdots, s_{t, N}\right\}$, and the production dynamic data table as auxiliary input is $S=\left\{S_{0}, S_{1}, \cdots, S_{T-1}\right\}$.

\subsection{Data Preprocessing}

3.2.1. Data Cleaning. During the production dynamic data recording process, due to well closure or human error recording, there will be some large floating points in the production dynamics. These data and normal production data are in different production conditions and should be eliminated. This time, using the segmented regression interpolation method,

\begin{tabular}{|c|c|c|c|c|}
\hline 1 & 2 & 3 & 4 & 5 \\
\hline 6 & 7 & 8 & 9 & 10 \\
\hline 11 & 12 & 13 & 14 & 15 \\
\hline 16 & 17 & 18 & 19 & 20 \\
\hline 21 & 22 & 23 & 24 & 25 \\
\hline
\end{tabular}

FIGURE 5: Schematic diagram of sampling points of saturation field data.

the 30-day data point is used as the fitting data to fit a straight line. When the true value is much greater than the fitting value, it is regarded as a noise point and the fitting value is used instead.

3.2.2. Data Standardization. By standardizing the input feature parameters, the problems of nonconvergence of the training process iterations and prediction errors due to the large difference in physical scale between the feature data can be alleviated, and the prediction accuracy is improved. The min-max standardization adopted this time, the original feature values are linearly transformed, and the data value range is transformed between $[0,1]$. The standardized minmax formula is as follows:

$$
x^{*}=\frac{x-x_{\min }}{x_{\max }-x_{\min }},
$$

where $x_{\max }$ is the maximum value of the sample data, $x_{\min }$ is the minimum value of the sample feature data, $x$ is the 
original feature value before normalization, and $x^{*}$ is the feature value after normalization.

The production dynamic data and saturation field data are normalized by columns.

3.2.3. Data Dimensionality Reduction. Because the auxiliary input saturation field data takes $5 \times 5$ data points around each well, the dimension of the data is large. If it is directly used as an input neuron, it will cause dimensional disaster and cause overfitting. Therefore, data dimensionality reduction is required. The author uses the PCA dimension reduction method to reduce the dimension $n$ of the original sample to the dimension $k(k<n)$ through a series of matrix transformations, that is, $k$-dimensional data can be used to represent most of the original $n$-dimensional information. The regularization method of PCA can effectively reduce the dimensionality and improve the convergence performance of the neural network gradient calculation process [47]. The steps are as follows:

Step 1. Standardize the data according to formula (3).

Step 2. Calculate the mean of all samples $\mu=(1 / n) \sum_{i=n}^{n} x_{i}$; $i$ represents the $i$ th feature. data.

Step 3. Calculate the covariance matrix of the sample

Step 4. Decompose the covariance matrix to obtain the eigenvector $u_{i}$ and eigenvalue $\lambda_{i}$.

Step 5. Determine the principal components and obtain new sample basis vectors.

Step 6. Transform the original data with a new basis vector to obtain new $k$-dimensional sample data.

3.2.4. Processed into Supervisory Data. The data of day $t$ and day $t+1$ of production dynamic data and saturation field data are used as input data $X$, and the data of day $t+2$ are used as output data $Y$, which is organized into a new data table. The purpose is to allow the deep neural network to better learn historical information and make the production dynamic prediction results more accurate.

3.2.5. Data Set Division. This time, $80 \%$ of the data is used as the training set to train the deep neural network, and $20 \%$ of the data is used as the verification set to verify the accuracy of the production dynamic prediction model.

3.3. Establish a Production Dynamic Prediction Model. Due to the small volume of oilfield production history data, it is impossible to use limited data training to obtain a predictive model with excellent performance, and oilfield production dynamics have phased and floating characteristics, and conventional neural network models are not suitable for establishing the production dynamic prediction model.

Based on the multi-input deep neural network structure proposed in Section 2.2, this paper uses production history data as the main input and oil saturation field data as the auxiliary input to establish a production dynamic prediction model specifically for oilfield production dynamic prediction. The use of the oil saturation field can comprehensively reflect the characteristics of the current oilfield production conditions and constrain the learning of the production history data by the learner, thereby improving the prediction accu- racy of the established production dynamic prediction model. Later, the production performance prediction model will be used to replace the conventional reservoir numerical simulator to calculate the future production performance of the randomly generated injection-production plan.

3.4. Generation and Prediction of Injection-Production Plan Based on Reservoir Engineering Constraints. In this step, the control frequency is used as a part of the injection-production parameter design, combined with reservoir engineering constraints, to generate different injection-production plans and then use the production dynamic prediction model formed in the previous step to predict the production dynamics and obtain the future production dynamic results of each plan.

The parameters to be designed for each plan are as follows: (1) control frequency; (2) water injection volume of each injection well during each regulation; (3) fluid production volume of each production well during each regulation.

Assuming that there are $N$ wells and $T$ time periods, the reservoir engineering constraints are as follows:

(1) Constraint of upper and lower fluid volume of a single well

$$
q^{\min }<q^{i}<q^{\max } \quad \forall i \in(0,1, \cdots, T-1) .
$$

For water injection wells, it needs to meet the following: minimum injection volume of single well $<$ water injection volume of injection well $<$ maximum injection volume of a single well; for production wells, it needs to meet the following: economic limit output of single well $<$ production well output $<$ maximum output of a single well.

(2) Constraint of total reservoir fluid production

$$
Q_{l, \min }<\sum_{j=1}^{N} \sum_{i=0}^{T-1} q_{l, j}^{i} \Delta t^{i}<Q_{l, \max }
$$

where $q_{l, j}^{i}$ is the fluid production of the $j$ th well in the $i$ th period, and $\Delta t^{i}$ is the $i$ th period. The total fluid production needs to be greater than the minimum total fluid production and less than the maximum total fluid production.

(3) Constraint of total reservoir injection volume

$$
\sum_{j=1}^{N} \sum_{i=0}^{T-1} q_{w, j}^{i} \Delta t^{i}=Q_{\text {const }}
$$

where $q_{w, j}^{i}$ is the water production of the $j$ th well in the $i$ th period, and the total water production needs to meet a fixed value. 
(4) Injection-production balance

$$
\sum_{j=1}^{N} \sum_{i=0}^{T-1} q_{l, j}^{i} \Delta t^{i}=\sum_{j=1}^{N} \sum_{i=0}^{T-1} q_{w, j}^{i} \Delta t^{i} .
$$

The total fluid production is equal to the total water injection. This option is optional and depends on the specific reservoir conditions.

The injection-production plan generated according to the above content is close to the actual production, and as the number of comparison plans increases, the control frequency of the optimal plan is close to the real optimal control frequency.

3.5. Comparison of Injection-Production Schemes Based on Objective Function. After using the production dynamic prediction model to predict the future production dynamics of the comparison scheme, the objective function value of each scheme can be calculated. The objective function value is used as the optimization criterion, and the scheme corresponding to the maximum objective function value is selected as the final optimization scheme.

From the perspective of reservoir management, oilfield development seeks to maximize production and maximize profits during the investment period. This paper takes the economic net present value (NPV) of oilfield development as the objective function. The larger the net present value, the better the injection-production plan and the better the investment benefit. The formula is as follows:

$$
\begin{aligned}
\mathrm{NPV}= & \sum_{i=1}^{n} \frac{p_{0} Q_{t}^{o}+p_{g} Q_{t}^{g}-p_{w}^{p} Q_{t}^{w \cdot p}+p_{w}^{i} Q_{t}^{w \cdot i}}{(1+r)^{t}} \\
& -\sum_{i=1}^{N}\left(C_{\mathrm{drill}} H_{i}+C_{c}\right),
\end{aligned}
$$

where NPV is the net present value, $\$$; $r$ is the discount rate, decimal; $n$ is the total production time, $\mathrm{d} ; N$ is the total number of wells; $C_{\text {drill }}$ is the average drilling cost per unit length, $\$ / \mathrm{m} ; H_{i}$ is the well depth of the $i$ th well, $\mathrm{m} ; C_{c}$ is the completion cost, $\$ /$ per port; $p_{0}$ is the crude oil sales price, $\$ / t ; p_{g}$ is the natural gas sales price, $\$ / \mathrm{m}^{3} ; Q_{t}^{o}$ is the crude oil sales volume on day $t, t ; Q_{t}^{g}$ is the total sales volume of natural gas on day $t, \mathrm{~m}^{3} ; p_{w}^{p}$ is the unit cost of water extraction, $\$ / t ; p_{w}^{i}$ is the unit water injection cost, $\$ / t ; Q_{t}^{w \cdot p}$ is the amount of water produced on day $t, t ; Q_{t}^{w \cdot i}$ is the amount of water injected on day $t, t$.

With the net present value (NPV) as the objective function, according to the historical production dynamic data and the predicted production dynamic data, the net present value of the generated injection-production plan at the end of the forecast period is calculated. Select the injectionproduction plan corresponding to the largest net present value as the optimal plan. The schematic diagram of the scheme optimization process is shown in Figure 6.

\section{Example Application}

4.1. Model Introduction. The actual reservoir model used in this paper is the L reservoir, which is a deep-water turbidite reservoir. There are two water injection wells and three production wells in the reservoir, which are driven by edge water. The model grid scale is $79 \times 109 \times 20$, the plane grid size is $50 \mathrm{~m} \times 50 \mathrm{~m}$, the average longitudinal grid step length is $10 \mathrm{~m}$, the average porosity is 0.17 , the average permeability is $446 \mathrm{mD}$, the oil saturation is 0.8 , and the average formation pressure is $49.2 \mathrm{MPa}$. The $\mathrm{L}$ reservoir has been in production for 225 days. The oil saturation field and well location are shown in Figure 7 . In order to make the flow field displacement more uniform and improve the oil recovery rate, the production system is optimized.

Obtain the daily water injection volume of each water injection well, the daily oil production and daily fluid production of each production well, and form the production dynamic input data table; obtain the daily oil saturation field value of the $5 \times 5$ grid around each well to form saturation. Enter the data table for the degree field.

4.2. Economic Parameters. Because oil reservoirs produce very little gas, natural gas prices are ignored. The total production cycle of the reservoir is 10 years (3650 days), and the net present value at the end of the production forecast cycle is used as the objective function. The parameter values of NPV in this article are shown in Table 1.

\subsection{Injection-Production Plan Generation Parameters.} According to the results of the reservoir engineering demonstration, the upper and lower bounds of the daily fluid production of a single well of oil production wells are $3000 \mathrm{~m}^{3} / \mathrm{D}$ and $0 \mathrm{~m}^{3} / \mathrm{D}$; the upper and lower bounds of the daily water injection of a water injection well are $4050 \mathrm{~m}^{3} / \mathrm{D}$ and $0 \mathrm{~m}^{3} / \mathrm{D}$, respectively. The reservoir with the optimized plan will be produced in the next 10 years (3650 days). Set the maximum control frequency in the next 10 years of production to 120 times. According to the above reservoir engineering constraints, 10,000 injection-production schemes are randomly generated.

4.4. Neural Network Training Parameters. Based on the multi-input neural network structure proposed in Section 2.2, ReLU is used as the activation function, and Adam is used as the optimizer. The number of neurons in the LSTM layer is 32 , the number of neurons in the fully connected layer 1 is 32 , and the number of neurons in the fully connected layer 2 is 64 . In the gradient descent process, the learning rate is 0.8 , the one-time training sample size is 30 , and the data is iteratively trained 5000 times. In order to avoid overfitting, the node discarding is introduced and the value is set to 0.2 .

4.5. Training Error Evaluation Method. This article uses mean relative error (MRE) and normalized root mean square 


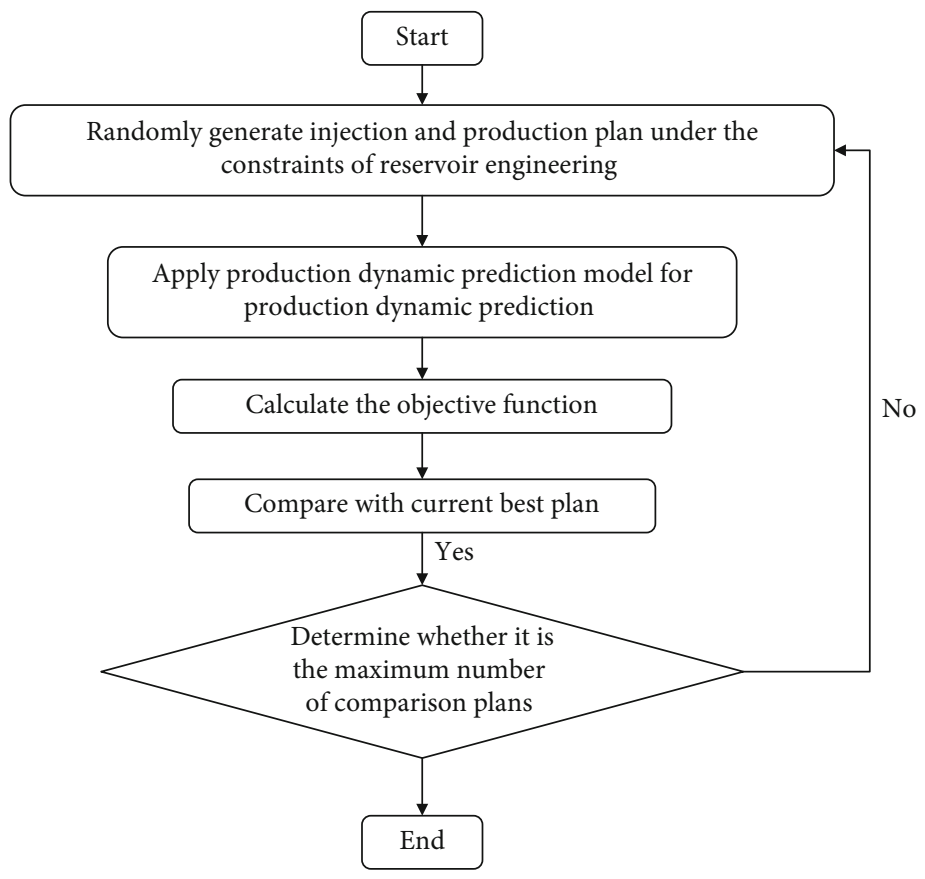

FIgURE 6: Scheme optimization flow chart.

error (nRMSE) as the evaluation criteria of training error. The specific calculation formula is as follows:

$$
\begin{gathered}
\operatorname{MRE}=\frac{1}{N} \sum_{i=1}^{N}\left|\frac{y_{i}^{\prime}-y_{i}}{y_{i}}\right|, \\
\mathrm{nRMSE}=\frac{1}{y_{\text {max }}-y_{\min }} \sqrt{\frac{1}{N} \sum_{i=1}^{N}\left(y_{i}^{\prime}-y_{i}\right)^{2} .}
\end{gathered}
$$

Among them, $N$ is the number of samples, $y_{i}$ is the predicted value of the $i$ th sample, $y_{\max }$ is the true value of the $i$ th sample, $y_{\min }$ is the maximum value of the sample, and the minimum value of the sample.

\subsection{Results and Discussion}

4.6.1. Comparison of Single Input and Multiple Input. The core of the well control optimization method proposed in this paper is to establish a production dynamic prediction model based on a multi-input deep neural network to replace the calculation of a reservoir numerical simulator, thereby improving calculation efficiency. The accuracy of the production dynamic prediction model determines whether the best solution can be selected from many injection and production solutions. Due to the phased characteristics of oilfield production and the large fluctuations of historical production data, the prediction results of the production dynamic prediction model obtained by previous scholars using the conventional LSTM neural network are not ideal. This paper proposes a multi-input neural network structure, which uses the oil saturation field to constrain the prediction of production dynamics to improve the accuracy of production dynamic prediction. In order to prove the effectiveness of this

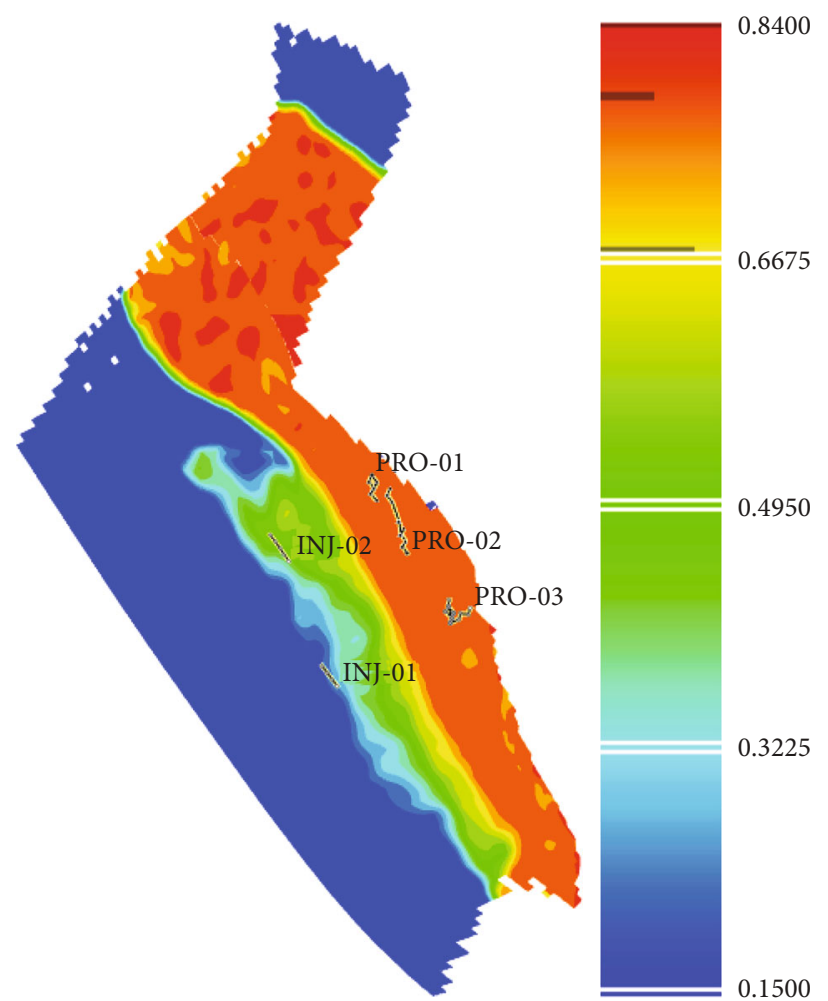

Figure 7: Oil saturation field of $\mathrm{L}$ reservoir.

method, this paper compares the prediction accuracy of the production dynamic prediction model based on the singleinput neural network structure of LSTM and the multiinput network structure. The single-input neural network structure only uses the production history data to train the 
TABLE 1: Net present value parameter values.

\begin{tabular}{lc}
\hline Economic parameter name (unit) & Value \\
\hline Crude oil prices $\left(\$ / \mathrm{m}^{3}\right)$ & 377 \\
Water injection price $\left(\$ / \mathrm{m}^{3}\right)$ & 20 \\
Water treatment fee $\left(\$ / \mathrm{m}^{3}\right)$ & 40 \\
Discount rate $(\%)$ & 10 \\
Drilling costs from the surface to the top of the reservoir $(\$ / \mathrm{m})$ & 515 \\
Drilling cost per meter inside the reservoir $(\$ / \mathrm{m})$ & 500 \\
\hline
\end{tabular}

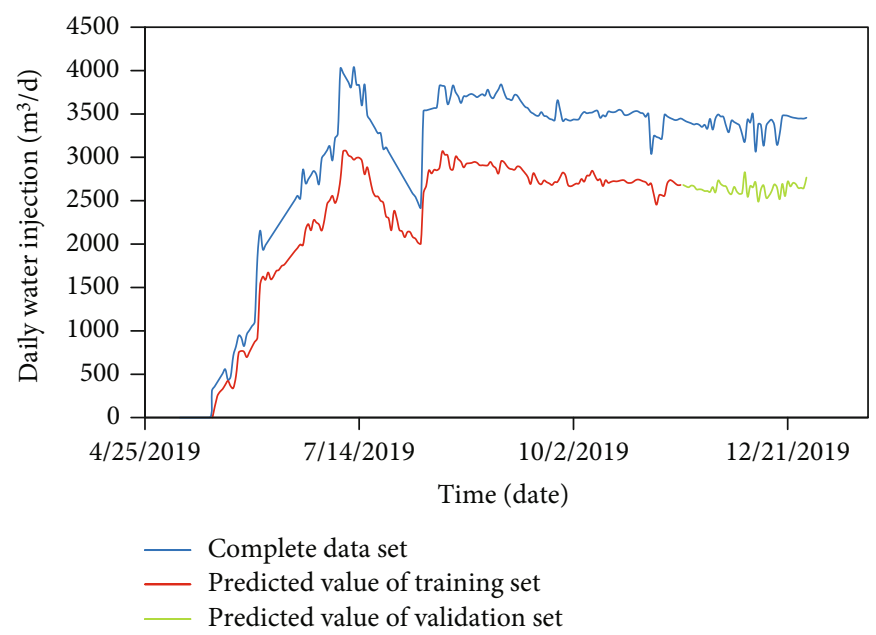

FIGURE 8: Forecast results of the single-input production dynamic forecasting model.

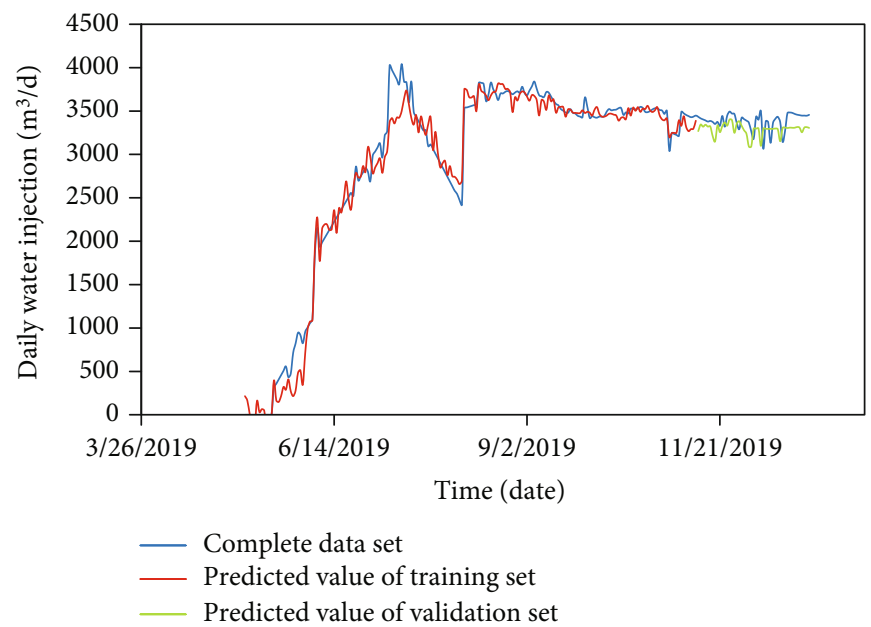

FIGURE 9: Forecast results of the multi-input production dynamic forecasting model.

neural network, while the multi-input neural network structure uses the production history data as the main input and the oil saturation field as the auxiliary input. Take the daily water injection volume of INJ-01 well as an example. The prediction results of the two are shown in Figures 8 and 9.

Compare the real daily water injection volume of INJ-01 in the verification set, the daily water injection volume prediction value of the single-input production dynamic prediction model of INJ-01, and the daily water injection volume prediction value of the multi-input production dynamic prediction model of INJ-01. The situation is shown in Table 2.

Comparing the single-input production performance prediction model and the multiple-input production performance prediction model in predicting the future production performance error of the reservoir, using MRE and nRMSE for evaluation, the results are shown in Table 3.

As can be seen from the table, compared with the singleinput production dynamic prediction model, the MRE of the 
TABLE 2: Comparison table of real value and predicted value of INJ-01 well.

\begin{tabular}{lccccc}
\hline $\begin{array}{l}\text { Time } \\
(\text { date })\end{array}$ & $\begin{array}{c}\text { Actual } \\
\text { value } \\
\left(\mathrm{m}^{3} / \mathrm{d}\right)\end{array}$ & $\begin{array}{c}\text { Single-input } \\
\text { predicted value } \\
\left(\mathrm{m}^{3} / \mathrm{d}\right)\end{array}$ & $\begin{array}{c}\text { Relative error of single-input } \\
\text { predicted value }(\text { decimal })\end{array}$ & $\begin{array}{c}\text { Multi-input } \\
\text { predicted value } \\
\left(\mathrm{m}^{3} / \mathrm{d}\right)\end{array}$ & $\begin{array}{c}\text { Relative error of multi-input } \\
\text { predicted value }(\text { decimal })\end{array}$ \\
\hline 2019.11 .12 & 1935.82 & 1553.48 & 0.1975 & 1867.03 & 0.0355 \\
2019.11 .20 & 1895.92 & 1514.15 & 0.2014 & 1925.83 & 0.0158 \\
2019.11 .28 & 1886.85 & 1571.58 & 0.1671 & 1890.38 & 0.0019 \\
2019.12 .6 & 1959.36 & 1400.08 & 0.2854 & 1836.93 & 0.0625 \\
2019.12 .14 & 1968.58 & 1509.8 & 0.2331 & 1927.92 & 0.0207 \\
2019.12 .22 & 1946.16 & 1553.86 & 0.2016 & 1942.29 & 0.002 \\
\hline
\end{tabular}

TABLE 3: MRE and nRMSE error results.

\begin{tabular}{lcc}
\hline Production dynamic prediction model & MRE & nRMSE \\
\hline Single-input & 0.1259 & 0.5983 \\
Multi-input & 0.0829 & 0.3665 \\
\hline
\end{tabular}

production dynamic prediction model based on the multiinput deep neural network is reduced by 0.043 , and the nRMSE is reduced by 0.2318 . It can be concluded that the multi-input deep neural network structure can improve the accuracy of the production dynamic prediction model.

4.6.2. Forecast Accuracy of Production Dynamics. In order to further explore the accuracy of the multi-input deep neural network for the production dynamic prediction, compare the water cut of the optimal solution under the numerical simulator and the water cut of the optimal solution under the production dynamic prediction model. And compare them with the water cut of the basic plan. The result is shown in Figure 10.

The results show that the water cut prediction based on the multi-input neural network production dynamic prediction model is basically the same as that of the traditional numerical simulator. Reservoir water cut is a comprehensive performance of reservoir production, so the optimized injection-production plan obtained under the production performance prediction model proposed in this paper is credible.

4.6.3. Comparison of Production Effects. The basic plan is to keep the production system at the last historical moment unchanged and continue to produce for 10 years. Taking the plan obtained through optimization of the production system optimization model as the optimization plan, the total daily liquid production and total daily water injection of the optimized plan are shown in Figure 11.

This article will compare the basic plan and the optimized plan from the following four aspects:

(1) Comparison of objective functions

Compare the NPV of the basic plan and the optimized plan in the next 10 years, as shown in Figure 12.

It can be seen from Figure 11 that in the next 10 years of reservoir water injection development, the NPV of the basic plan is $1.81 \times 10^{9} \$$, the net present value of the optimized plan is $1.98 \times 10^{9} \$$, and the optimized plan has increased by $1.7 \times 10^{8} \$$ compared to the basic plan NPV.

(2) Comparison of cumulative oil production and cumulative water production

It can be seen from Figure 13 that the cumulative oil production of the optimized plan increased by $103.8 \times 10^{4} \mathrm{~m}^{3}$ compared to the basic plan, and the cumulative water production decreased by $108.4 \times 10^{4} \mathrm{~m}^{3}$.

(3) Remaining oil distribution map

It can be seen from Figure 14 that the remaining oil contour of the optimized plan is flatter than the basic plan, the displacement is more uniform, and the saturation of the remaining oil is significantly reduced near the production well, especially in the vicinity of the PRO-03 well, indicating the optimization. The plan can improve the effect of oilfield development.

\section{(4) Recovery factor}

It can be seen from Figure 15 that the optimized plan has increased the recovery factor by $2.76 \%$ after 10 years of reservoir development compared with the basic plan, which has promoted the development effect of the oilfield.

4.6.4. Optimization Time Comparison. This L model's production system optimization compares 10,000 plans, which takes a total of 4.2 hours. The average time for a single plan to predict 10 -year production dynamics is $1.5 \mathrm{~s}$. Regardless of whether the predecessor optimization based on reservoir numerical simulation or optimization theory is used, if you want to compare 10,000 plans, you need at least $10,000 \times$ $0.8 \mathrm{~h}=8000 \mathrm{~h}$. In summary, the use of a deep neural network-based production system optimization method greatly improves the speed of optimization and reduces the consumption of manpower and material resources.

4.6.5. Control Frequency. Because this method can quickly generate thousands of injection-production plans and can quickly optimize these plans, it is entirely possible to use this advantage to control the frequency as part of the injectionproduction parameter design. As the number of comparison schemes continues to increase, the control frequency of the obtained optimal scheme is closer to the real optimal control 


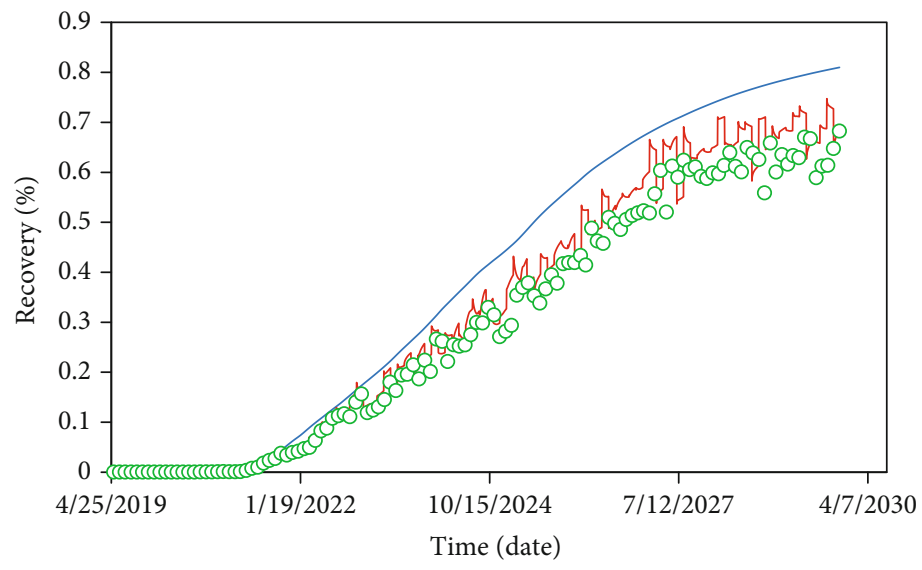

Basic plan

Optimization plan (numerical simulator)

- Optimization plan (neural network)

FIGURE 10: Comparison of water content curves.

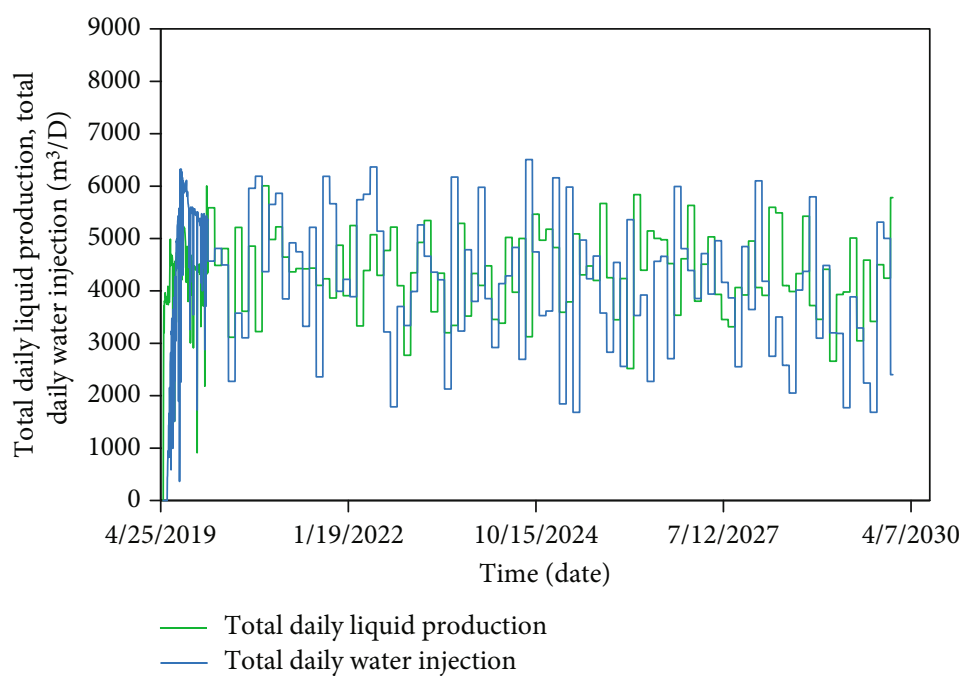

Figure 11: Production system with the optimized plan.

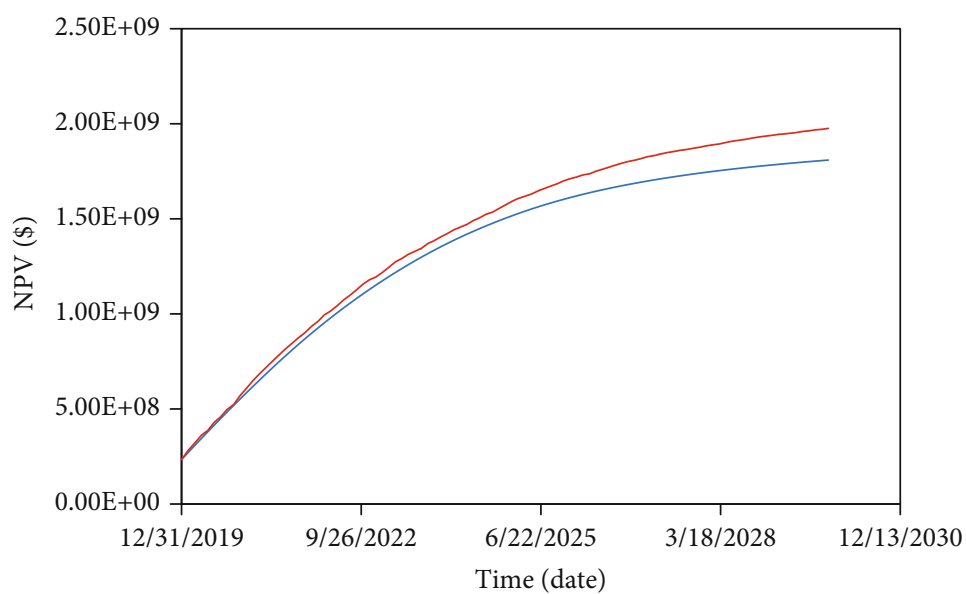

NPV of the basic plan

Optimization plan NPV

FIGURE 12: NPV comparison in the next 10 years. 


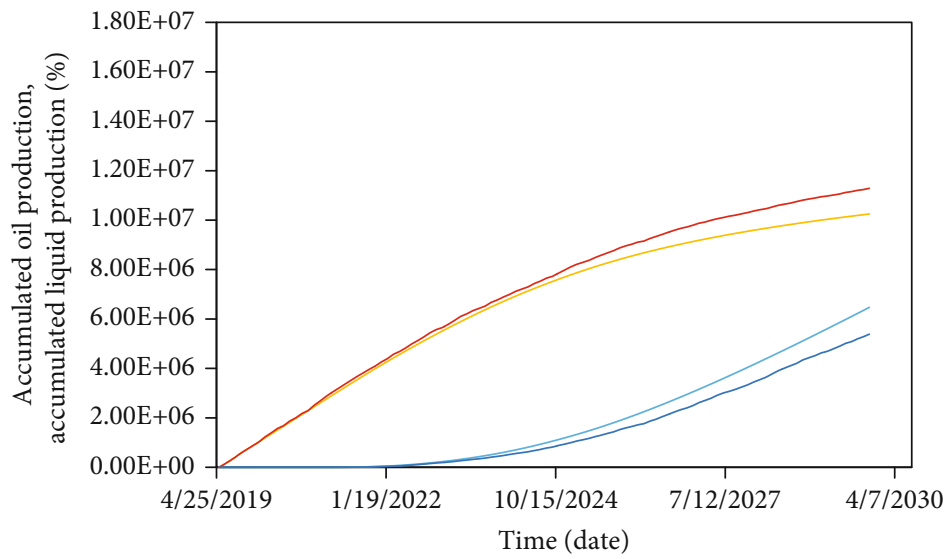

_ Accumulated oil production of basic plan

Accumulated liquid production of basic plan

- Accumulated oil production of the optimization plan

- Accumulated water production of the optimization plan

FIGURE 13: Comparison of accumulated oil and accumulated water.

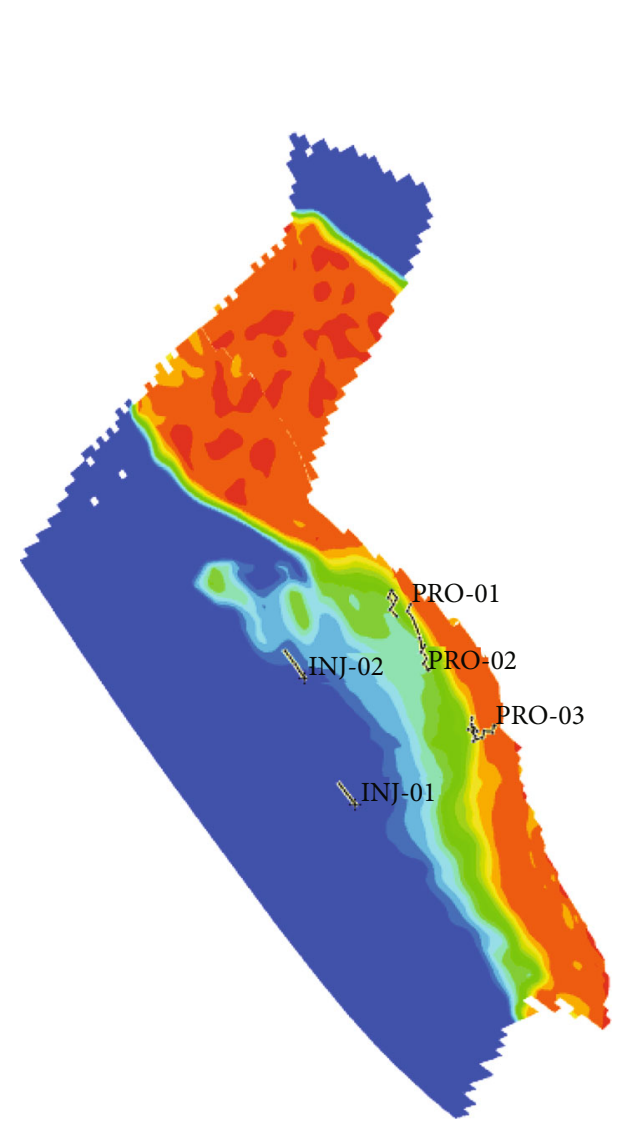

(a)

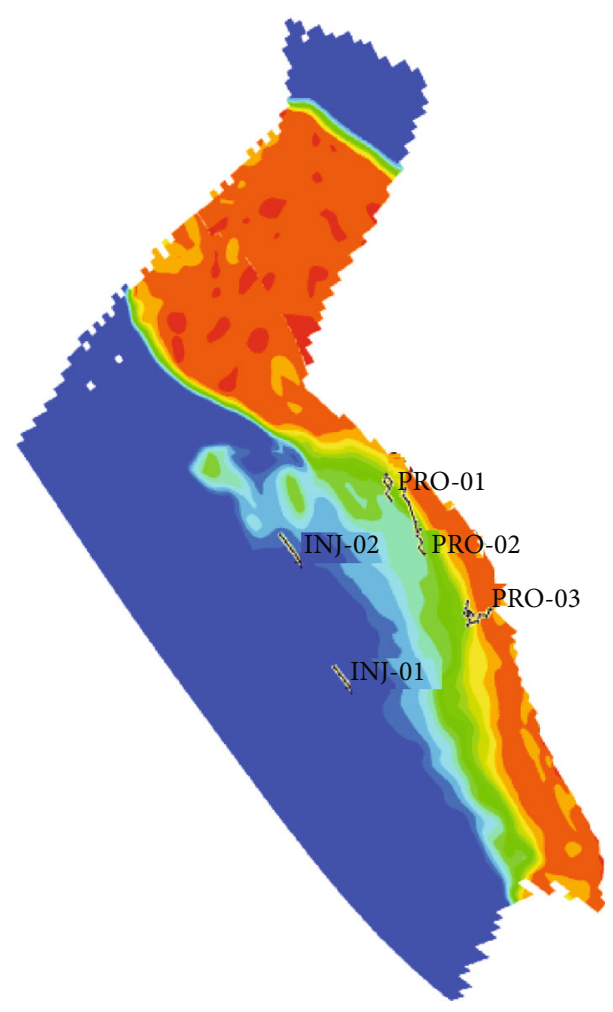

(b)
Saturation of oil, Dimensionless quantity,Avg

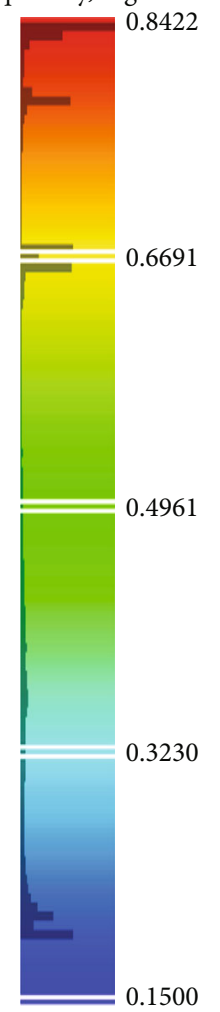

Figure 14: Comparison chart of the remaining oil saturation contour. 


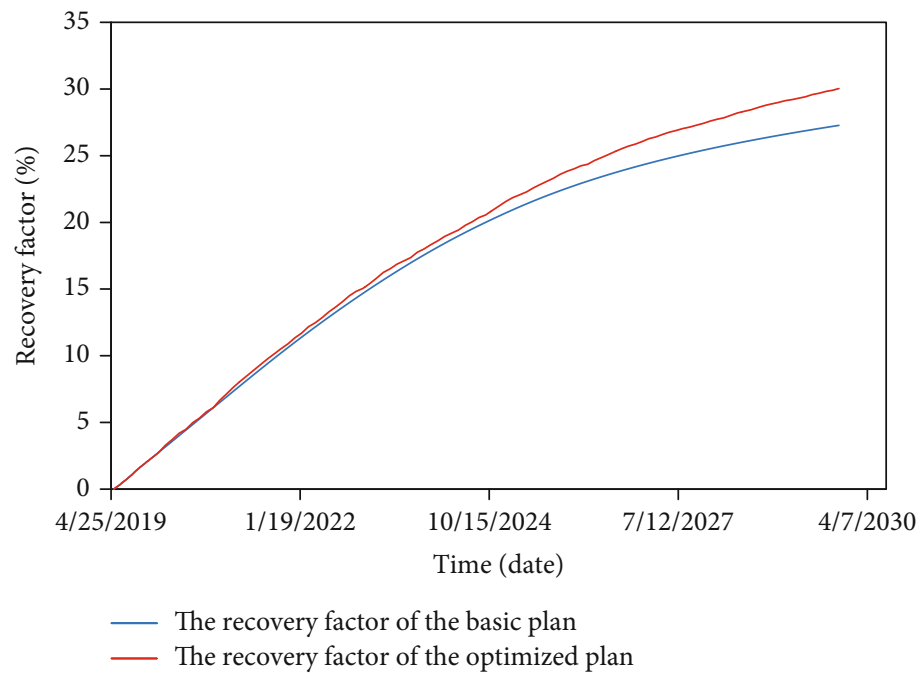

Figure 15: Comparison of recovery factor.

frequency, and the search for the optimal control frequency is realized to a certain extent. After comparing the 10,000 programs, the optimal control frequency of the optimal program is once every 36 days, and a total of 101 times in 10 years.

\section{Summary}

(1) The core of this new method of a well control optimization method proposed in this paper is to establish a production dynamic prediction model based on a multi-input neural network. In order to solve the problem that the LSTM neural network cannot accurately predict the characteristics of the reservoir production dynamics in stages and large changes, this paper proposes a neural network structure based on multiple inputs. With production dynamic data as the main input, oil saturation field as the auxiliary input, and LSTM, the production dynamic prediction model has high accuracy and conforms to the actual situation of reservoir production

(2) The optimization speed of the production system optimization method based on a deep neural network is extremely fast, because it gets rid of the limitation of conventional reservoir numerical simulation software, and does not require engineers to preselect the plan. It only needs to use the algorithm to give each parameter under the reservoir engineering constraints. With different values, tens of thousands of solutions can be combined, and the optimal solution can be quickly found out after optimization of production dynamics. A large number of scheme comparisons ensure that the optimization scheme is close to the true optimal scheme

(3) With the help of the characteristics of rapid optimization, the control frequency can be used as the optimization parameter. After a large number of injectionproduction schemes are compared and optimized, the control frequency of the optimized plan is close to the optimal control frequency in the true sense. And it is determined that the optimal control frequency of the $\mathrm{L}$ reservoir in the next ten years is 101 times

(4) The verification of the example shows that this method achieves an increase in the NPV of the L reservoir after ten years of development. The NPV increases by $1.7 \times 10^{8} \$$, the water cut decreases, the cumulative oil production increases by $103.8 \times 10^{4}$ $\mathrm{m}^{3}$, and the cumulative water production decreases by $108.4 \times 10^{4} \mathrm{~m}^{3}$. The recovery factor has been improved by $2.76 \%$ and other excellent indicators, proving that the optimization method of the production system based on a deep neural network can improve the recovery and economic benefits of oilfield development

\section{Data Availability}

The data used to support the findings of this study are available from the corresponding author upon request.

\section{Conflicts of Interest}

The authors declare that they have no conflicts of interest.

\section{Acknowledgments}

This research was supported by the National Science and Technology of Major Project (Grant nos. 33550000-19FW1701-0074 and 2016ZX05010006-006). The authors also wish to appreciate the State Key Laboratory of Petroleum Resources and Prospecting, China University of Petroleum (Beijing) for the permission to publish this paper. 


\section{References}

[1] D. R. Brouwer and J. D. Jansen, "Dynamic optimization of waterflooding with smart wells using optimal control theory," SPE Journal, vol. 9, no. 4, pp. 391-402, 2004.

[2] L. Wenfu, "Optimizing and adjusting method of the waterflooding injection-production structure at extra-high watercut stage for Daqing Sanan Oilfield," Daqing Petroleum Geology and Development, vol. 39, no. 4, pp. 53-58, 2020.

[3] C. Huijiang, S. Gangyi, C. Xiaoming, Z. Shangqi, and Z. Yanhui, "Lateral injection - production optimization and application based on balanced flooding," Special Oil and Gas Reservoir, vol. 26, no. 4, pp. 120-124, 2019.

[4] H. Chang, Y. Liu, Y. Lei, and Q. Zhang, "A comprehensive workflow for real time injection-production optimization based on equilibrium displacement," Advances in Geo-Energy Research, vol. 4, no. 3, pp. 260-270, 2020.

[5] H. U. Dan-Dan, M. S. Feng, W. U. Yuan-Bing, H. U. SongHao, and Y. P. Luo, "Suitability analysis and injectionproduction optimization of $\mathrm{co} 2$ miscible flooding to carbonate reservoirs in Abu Dhabi's onshore giant oilfields," Science Technology and Engineering, vol. 15, no. 3, pp. 84-89, 2015.

[6] D. U. DianFa, B. A. Zhongchen, L. Yang, W. Weilin, and L. Gang, "Optimization of injection production parameters for activated waterflooding in heavy oil reservoir," Special Oil and Gas Reservoir, vol. 21, no. 1, 2014.

[7] L. Xiangbin, W. Xianjun, W. Haijing et al., "The physical simulation and optimization of injection-production parameters in combined thermal carrier steam stimulation," Special Oil and Gas Reservoir, vol. 21, no. 3, pp. 135-137, 2014.

[8] N. Fang, Z. N. Zhang, M. J. Cheng, Z. Q. Zhu, and Q. Cheng, "Injection-production parameter optimization study of cyclic water injection at different development levels of fractures," Special Oil and Gas Reservoir, vol. 26, no. 1, pp. 131-135, 2019.

[9] L. Chun, X. Shuang, and D. Yang, "Optimization of injection mode and injection parameters in multi - well fracture-cavity units of Tahe," Journal of Chongqing University of Science and Technology, vol. 16, no. 3, pp. 44-47, 2014.

[10] W. U. Jie, Study on Remaining Oil Distribution by Waterflooding Process and Optimization of Parameters in Daluhu Oilfield, China University of Petroleum, Beijing, 2017.

[11] X. Xiaoqing, "A new algorithm for non-gradient optimization of injection-production parameters for polymer flooding," Lithologic Reservoirs, vol. 31, no. 31, pp. 139-146, 2019.

[12] L. Fan, Z. Wensheng, S. Jian, W. Kai, and Z. Kai, “Optimization methods of development and production control in water drive oilfield," Petroleum Geology and Engineering, vol. 32, no. 4, pp. 69-71, 2018.

[13] Z. H. Hui, T. A. Yiwei, and K. A. Zhijiang, "Reservoir production optimization using an upgraded perturbation gradient approximation algorithm," Journal of China University of Petroleum, vol. 40, no. 2, pp. 99-104, 2016.

[14] Z. Kai, Y. Jun, Z. Liming, L. Qin, and Q. Huanhuan, "Injectionproduction strategies optimization for efficient development of water flooding," Systems Engineering-Theory \& Practice, vol. 30, no. 8, pp. 1506-1513, 2010.

[15] Z. Fengjiu, "Optimization method of injection and production parameters for polymer / surfactant binary flooding," Journal of China University of Petroleum, vol. 42, no. 5, pp. 98-104, 2018.

[16] Y. Xia, L. Yang, and Y. Jun, "Reservoir production optimization method based on modified simplex gradient algorithm,"
Petroleum Geology and Recovery Efficiency, vol. 20, no. 3, pp. 65-67, 2013.

[17] Z. Kan, Seepage Mechanisms and Development Optimization of Preformed Particle Gel Flooding, China University of Petroleum, Beijing, 2017.

[18] P. Lian, L. Li, and T. Duan, "Injection parameters optimization of crosslinked polymer flooding by genetic algorithm," Advances in Geo-Energy Research, vol. 2, no. 4, pp. 441-449, 2018.

[19] J. Fu and X. H. Wen, "Model-based multiobjective optimization methods for efficient management of subsurface flow," SPE Reservoir Simulation Conference, vol. 22, no. 6, 2017.

[20] J. Fu and X. H. Wen, "An assessment of model-based multiobjective optimization for efficient management of subsurface flow," in SPE Western Regional Meeting, pp. 1-27, Garden Grove, CA, USA, 2018.

[21] A. Yazdanpanah, A. Rezaei, H. Mahdiyar, and A. Kalantariasl, "Development of an efficient hybrid GA-PSO approach applicable for well placement optimization," Advances in GeoEnergy Research, vol. 3, no. 4, pp. 365-374, 2019.

[22] S. Wang, Z. Li, S. Wang, and X. Han, "Well pattern optimization based on StoSAG algorithm," Advances in Geo-Energy Research, vol. 2, no. 1, pp. 103-112, 2018.

[23] M. A. Ahmadi, "Connectionist approach estimates gas-oil relative permeability in petroleum reservoirs: application to reservoir simulation,” Fuel, vol. 140, pp. 429-439, 2015.

[24] K. Lee, S. Kim, J. Choe, B. Min, and H. S. Lee, "Iterative static modeling of channelized reservoirs using history-matched facies probability data and rejection of training image," Petroleum Science, vol. 16, no. 1, pp. 127-147, 2019.

[25] K. Lee, J. Lim, S. Ahn, and J. Kim, "Feature extraction using a deep learning algorithm for uncertainty quantification of channelized reservoirs," Journal of Petroleum Science and Engineering, vol. 171, no. 12, pp. 1007-1022, 2018.

[26] H. I. Asala, J. Chebeir, W. Zhu, I. Gupta, A. D. Taleghani, and J. Romagnoli, "A machine learning approach to optimize shale gas supply chain networks," in SPE Annual Technical Conference and Exhibition, pp. 1-29, San Antonio, TX, USA, 2017.

[27] J. Sun, X. Ma, and M. Kazi, "Comparison of decline curve analysis DCA with recursive neural networks RNN for production forecast of multiple wells," in SPE Western Regional Meeting, pp. 1-11, Garden Grove, CA, USA, 2018.

[28] K. Lee, J. Lim, D. Yoon, and H. Jung, "Prediction of shale-gas production at Duvernay formation using deep-learning algorithm," SPE Journal, vol. 24, no. 6, pp. 2423-2437, 2019.

[29] Y. Li, R. Sun, and R. Horne, "Deep learning for well data history analysis," in SPE Annual Technical Conference and Exhibition, pp. 1-16, Calgary, AB, Canada, 2019.

[30] C. Yuanqian, "Derivation and application of Weng's prediction model," Natural Gas Industry, vol. 2, pp. 22-26, 1996.

[31] J. Guo, Research on the technology policy of horizontal well development in gas fields, China University of Geosciences, 2012.

[32] Z. Yanyan, Y. Xiangchun, and K. Zhijiang, "The production and pressure change model of fractured-vuggy carbonate reservoirs," Oil and Gas Geology, vol. 31, no. 1, pp. 54-56, 2010.

[33] H. Guangqing, "Production decline analysis and decline rate characterization formula during ultra-high water cut period," Science Technology and Engineering, vol. 19, no. 15, pp. 99$104,2019$. 
[34] J. Wang, S. Chengfang, J. Shuhong, L. Guanlin, and C. Yingqiao, "New type of water drive characteristic curve in the ultra-high water cut period," Petroleum Exploration and Development, vol. 44, no. 6, pp. 955-960, 2017.

[35] R. Yanlong, G. Jianwei, C. Wenfu, and Z. Yigen, “Oilfield production prediction model based on improved fruit fly algorithm and long short-term memory neural network," Science Technology and Engineering, vol. 20, no. 18, pp. 7245-7251, 2020.

[36] J. Lu, "Prediction method of annual oil production and water cut in oilfields," Daqing Petroleum Geology and Development, vol. 4, pp. 62-65, 2007.

[37] C. Wang, "Research on data mining algorithm based on neural network," Modern Electronic Technology, vol. 40, no. 11, pp. 111-114, 2017.

[38] T. Li, Y. Shuo, and Y. Wei, "Research on model building and overfitting of deep learning," Computer Times, no. 2, pp. 14$17,2018$.

[39] Y. Mao, "Long text emotion classification method based on attention double layer LSTM," Journal of Chongqing Electronic Engineering Vocational College, vol. 28, no. 2, pp. 118-125, 2019.

[40] Y. Peng, X. Liu, W. Wang, X. Zhao, and M. Wei, "Image caption model of double LSTM with scene factors," Image and Vision Computing, vol. 86, no. 6, pp. 38-44, 2019.

[41] S. Hochreiter and J. Schmidhuber, "Long short-term memory,” Neural Computation, vol. 9, no. 8, pp. 1735-1780, 1997.

[42] Z. Ruizhen, H. Yueping, and Z. Xiaotong, "End-to-end speech recognition based on deep LSTM," Journal of North University of China (Natural Science Edition), vol. 191, no. 3, pp. 58-62, 2020.

[43] W. Shihao, Z. Qinzheng, Y. Han, L. Qianmu, and Q. Yong, “A network traffic prediction method based on LSTM[J]," ZTE Communications, vol. 17, 2019.

[44] J. Wang, C. Junxing, L. Zhekui, Z. Xin, and L. Xue, "Predrilling log curve prediction method based on long and short-term memory network," Journal of Chengdu University of Technology (Natural Science Edition), vol. 47, no. 2, 2020.

[45] S. Hochreiter, "The vanishing gradient problem during learning recurrent neural nets and problem solutions," International Journal of Uncertainty, Fuzziness and KnowledgeBased Systems, vol. 6, no. 2, pp. 107-116, 2011.

[46] N. Srivastava, G. Hinton, A. Krizhevsky, I. Sutskever, and R. Salakhutdinov, "Dropout: a simple way to prevent neural networks from overfitting," Journal of Machine Learning Research, vol. 15, no. 1, pp. 1929-1958, 2014.

[47] J. Fu and X.-H. Wen, "A regularized production-optimization method for improved reservoir management," SPE Journal, vol. 23, no. 2, pp. 467-481, 2018. 